\title{
CLINICAL PRACTICE Exercises in Clinical Reasoning
}

\section{A Missed Opportunity}

\author{
Ryan Van Wert, $M D^{7}$, Ann Marie McKenna, $M D^{7}$, Christopher A. Smith, $M D^{2}$, \\ Peter Vadas, MD, $P h D^{7}$, Allan S. Detsky, MD, PhD ${ }^{3,4}$, and Ophyr Mourad, MD, MSc ${ }^{7}$ \\ 'Department of Medicine, St. Michael's Hospital, University of Toronto, Toronto, ON, Canada; ${ }^{2}$ Department of Medicine, Queens University, \\ Kingston, ON, Canada; ${ }^{3}$ Department of Medicine, St. Michael's Hospital, University of Toronto and University Health Network, Toronto, ON, \\ Canada; ${ }^{4}$ Department of Medicine and Health Policy Management and Evaluation, Mount Sinai Hospital, Toronto, ON, Canada.
}

KEY WORDS: medical education; cognition; problem solving; infectious disease; immunology.

J Gen Intern Med 28(5):728-32

DOI: $10.1007 / \mathrm{s} 11606-012-2248-5$

(c) Society of General Internal Medicine 2012

\section{CLINICAL INFORMATION}

A 42-year-old healthy male carpenter presented to a new family doctor for a periodic health examination. He described a chronic, non-productive cough of 5 years duration. He reported no other previous health problems. Physical examination was normal. A complete blood count revealed a hemoglobin of $164 \mathrm{~g} / \mathrm{L}$, a platelet count of $282 \times 10^{9}$ cells/L, and a total WBC count of $6.0 \times 10^{9}$ cells $/ L$ with a differential neutrophil count of 5.0 $\times 10^{9}$ cells $/ L$ (normal range 4.0-11.0 $\times 10^{9}$ cells/L) and lymphocyte count of $0.3 \times 10^{9}$ cells/L (normal range 1.0-3.2 $\times 10^{9}$ cells $/ L$ ). A chest radiograph revealed hilar adenopathy with perihilar reticulonodular infiltrates. Pulmonary function testing was reported as normal. Bronchoscopy with endobronchial biopsies revealed non-caseating granulomas and a diagnosis of Stage II sarcoidosis was made. In the following month, his respiratory symptoms worsened and he was treated with oral glucocorticoids (prednisone $0.5 \mathrm{mg} / \mathrm{kg} / \mathrm{day}$ for 1 month, followed by $0.25 \mathrm{mg} / \mathrm{kg} /$ day for 5 months). His respiratory symptoms gradually improved, but toward the end of therapy new onset headaches and photophobia developed.

\section{CLINICIAN}

This patients' presentation with chronic cough, bilateral hilar adenopathy, reticulonodular infiltrates, and lymphopenia, followed by biopsy confirmation of non-caseating granulomas, make sarcoidosis a reasonable initial working diagnosis. Despite the typical clinical, radiological and

Received May 22, 2012

Revised September 13, 2012

Accepted September 26, 2012

Published online November 13, 2012 histological findings, and the fact that lymphopenia is known to occur in half of patients, sarcoidosis remains a diagnosis of exclusion. It should also be remembered that non-caseating granulomas are not pathognomonic of sarcoidosis and are also associated with a multitude of other conditions, including fungal, neoplastic and connective tissue diseases. The new onset headache and photophobia while on prednisone is unexpected. Although Sarcoidosis affects the neurologic system in 5-15\% of patients, cranial nerve palsies are the most common manifestation, and the majority of patients with neurosarcoidosis present with neurological symptoms as their first manifestation. Alternative diagnoses such as tuberculosis (TB), neurosyphilis, Brucellosis, Lyme disease, Whipple's disease, autoimmune disease and malignancy, including leptomeningeal carcinomatosis, must be first excluded before a diagnosis of neurosarcoidosis is made. As this patient was also on prednisone, further workup, including cerebrospinal fluid (CSF) analysis and neuroimaging, is required in order to exclude opportunistic central nervous system infection.

\section{DIAGNOSTIC REASONING}

The discussant describes a problem representation of chronic cough, hilar adenopathy and non-caseating granulomas. This current "pattern recognition" is consistent with the working diagnosis of sarcoidosis. Further diagnostic hypotheses will be influenced by features that should be present or should be absent in this condition. For example, atypical features on neuro-imaging or the lumbar puncture should lead to hypothesis modification and refinement.

Magnetic resonance imaging of the brain showed abnormal leptomeningeal enhancement (Fig. 1). A lumbar puncture was performed and appeared clear. Cerebrospinal fluid total white cell count was $127 \times 10^{6} / \mathrm{L}$, with a differential count of $51 \%$ monocytes and $36 \%$ neutrophils. Cerebrospinal fluid glucose was low at $1.4 \mathrm{mmol} / \mathrm{L}$ (normal range $2.8-4.4 \mathrm{mmol} / \mathrm{L}$ ) and $\mathrm{CSF}$ protein was elevated at $1.61 \mathrm{~g} / \mathrm{L}$ (normal range $0.12-0.60 \mathrm{~g} / \mathrm{L}$ ). Gram stain and culture were negative, 

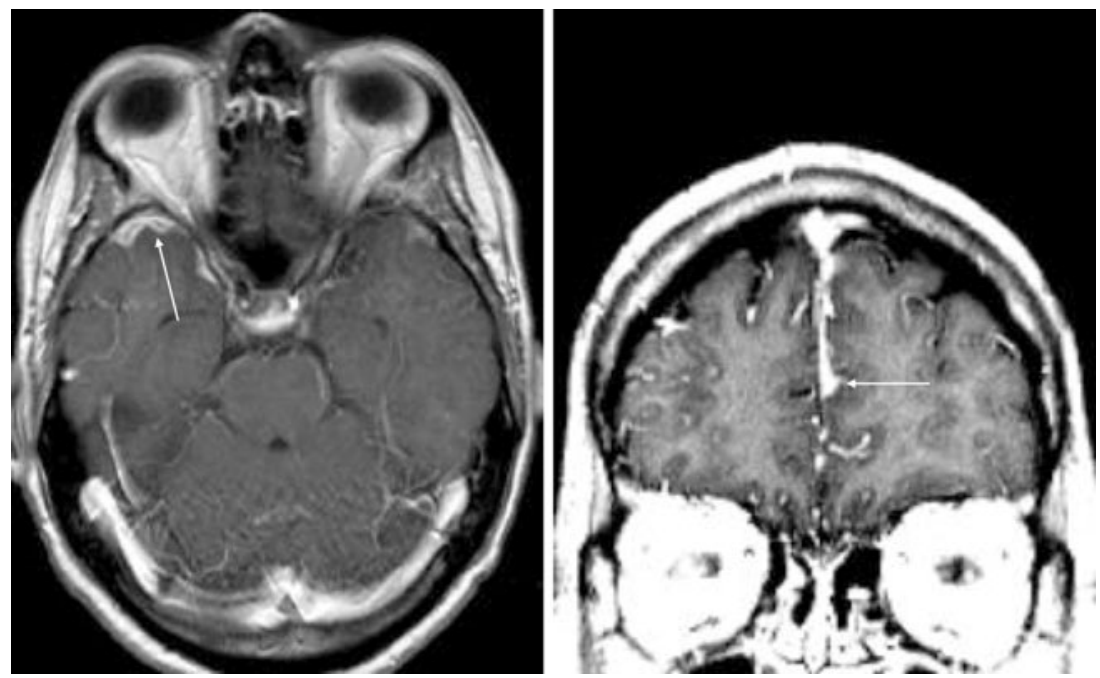

Figure 1. Selected magnetic resonance imaging (MRI) axial and coronal post contrast T1-weighted images showing abnormal leptomeningeal enhancement (arrows).

acid-fast bacilli were not seen, and cytology was negative for malignant cells. A diagnosis of neurosarcoidosis was made. Glucocorticoid therapy was intensified and methotrexate was added.

The finding of leptomeningeal enhancement is nonspecific and possibilities include meningitis or lymphoma, as well as neurosarcoidosis. The diagnosis of neurosarcoidosis is challenging because of the requirement of pathology for definitive diagnosis, and because malignancy, demyelinating disease, other autoimmune disease and various infections are often important diagnostic considerations. The lumbar puncture results are intriguing, as they do not show the typical findings of neurosarcoidosis, which include a lymphocytic pleocytosis and normal CSF glucose. In fact, the monocytic pleocytosis, (if confirmed), is very unusual. The low glucose, although described in neurosarcoidosis, is more commonly associated with bacterial, mycobacterial or fungal central nervous system infections. The negative acid-fast bacilli and cytology findings are not completely reassuring, as they have low sensitivity. In light of these discrepancies, I would be hesitant to accept the working diagnosis of neurosarcoidosis until other possibilities have been excluded.

The headache recurred within 2 weeks and repeat lumbar puncture identified many budding yeast organisms. Fungal culture grew Cryptococcus neoformans. Immunosuppressive therapy was stopped, serial lumbar punctures were performed, and liposomal amphotericin $B$ and flucytosine were initiated. Human Immunodeficiency Virus (HIV) antibody testing was negative.

When this patient, with a history, radiological and histological findings suggestive of sarcoidosis, presented with headache and photophobia, a presumptive diagnosis of neurosarcoidosis was made. Unfortunately, no neuro- diagnostic tests are pathognomonic for neurosarcoidosis and the CSF findings in this patient are not typical. Premature closure (the failure to consider other diagnoses after an apparent solution has been found) is the most likely explanation for what happened cognitively. In addition, failure to adequately adjust or consider alternate diagnoses in the light of new data and remaining fixed to one's original hypothesis (anchoring and adjustment bias) are other explanations. Now that Cryptococcal meningitis has been diagnosed, it is imperative for the clinician to adjust and refine his or her diagnostic reasoning.

Although now most commonly associated with HIV infection and as an AIDS defining illness, other risk factors for Cryptococcal meningoencephalitis include any condition that can impair the cell-mediated immune system. Examples include immunosuppressive therapy (such as prednisone), hematological malignancies, connective tissue diseases and sarcoidosis. The importance of pursuing and correctly identifying an underlying condition is critical, as further treatment with immunosuppressive therapy could be detrimental.

One month later, a bacterial pneumonia was successfully treated and 2 months later a deep vein thrombosis (DVT) was diagnosed. Four months after being hospitalized with cryptococcal meningoencephalitis, the patient reported a 1 day history of neck pain, voice changes, sore throat, headache and fever. Physical examination was remarkable for a blood pressure of 70/33, heart rate 134 beats per minute (sinus tachycardia), a respiratory rate of 14 , a temperature of $36.9^{\circ} \mathrm{C}$, and oxygen saturation was $98 \%$ on $2 \mathrm{~L}$ of oxygen by nasal prongs. The oropharynx was clear and cranial nerves III-VIII and XII were intact. The precordial, respiratory and abdominal examinations were normal. Medications included fluconazole $400 \mathrm{mg}$ po daily, 
piperacillin-tazobactam 4.5 g IV TID, hydrocortisone $100 \mathrm{mg}$ IV q8h, and enoxaparin $120 \mathrm{mg}$ sc daily. Blood work revealed a Hemoglobin of $103 \mathrm{~g} / \mathrm{L}$, a platelet count of a $106 \times 10^{9}$ cells $/ L$, and a total WBC count of $3.43 \times$ $10^{9}$ cells $/ \mathbf{L m}$ with a differential neutrophil count of $1.784 \times 10^{9}$ cells $/ L$ (normal range $4.0-11.0 \times 10^{9}$ cells $/ L$ ), and lymphocyte count of $0.069 \times 10^{9}$ cells/L (normal range 1.0-3.2 $\times 10^{9}$ cells/L). HIV-1 and 2 antibody testing were again negative, as were serology for Hepatitis B and C. A two-dimensional echocardiogram revealed absence of a pericardial effusion. Computed tomography (CT) of the neck was suggestive of retropharyngeal space infection (Figure 2), and blood cultures grew Streptococcus pyogenes. He was treated with Penicillin G 2million units IV six times daily and recovered.

He has had multiple recent infections, (pneumonia, meningitis and now retropharyngeal infection with sepsis), lymphopenia, and a new pancytopenia with known noncaseating granulomas. The pancytopenia could be related to his sepsis, or may indicate bone marrow involvement. Hematological malignancy, other infections, (including acute HIV), congenital immunodeficiency states, and autoimmune diseases should be ruled out. I would ask for a reticulocyte count and peripheral smear with flow cytometry. I'd also request an HIV p24 antigen, anti-treponemal testing for syphilis, Epstein-Barr virus and Cytomegalovirus (CMV) serology, plus antinuclear antibody (ANA) and antineutrophil cytoplasmic antibody (ANCA) serologies. Measurement of serum immunoglobulins (IgG, $\operatorname{IgA}, \operatorname{IgM}$ ) should be done. If these investigations are nondiagnostic, then I would consider a bone marrow biopsy.

Diagnostic reasoning in complex cases requires that the clinician systematically refine or modify hypotheses and eliminate competing ones. The problem presentation is now a 42-year-old man with recurrent infections, lymphopenia and biopsy evidence of non-caseating granulomas. The clinician has moved to an analytical approach to generate a broad differential diagnosis and systematically evaluate the gathered data and sequentially eliminate certain hypotheses. Here, the discussant admits to a lack of certainty about the diagnosis. The three major categories of undefined systemic illnesses (infection, malignancy and autoimmune) all remain in play.

The patient improved and was transferred out of the intensive care unit. Repeat complete blood count again revealed a lymphopenia $\left(0.230 \times 10^{9}\right.$ cells/L) and an absolute CD4 count of $55 \times 10^{6} /$ cells L. HIV-1 p24 Antigen screen was non-reactive. Albumin and total protein were both low at 14 and $53 \mathrm{~g} / \mathrm{L}$ respectively. Antinuclear and antineutrophil cytoplasmic antibodies were negative. Urine microscopy did not reveal any active sediment. A bone marrow aspirate revealed a normocellular specimen with a moderate reduction in lymphocytes, and biopsy revealed normal trilineage hematopoiesis. Flow cytometry of peripheral blood was consistent with marked lymphopenia, but no evidence of clonal expansion. A proportionate reduction of $\mathrm{CD3}+\mathrm{CD} 4+$ and $\mathrm{CD3}+\mathrm{CD8}+$
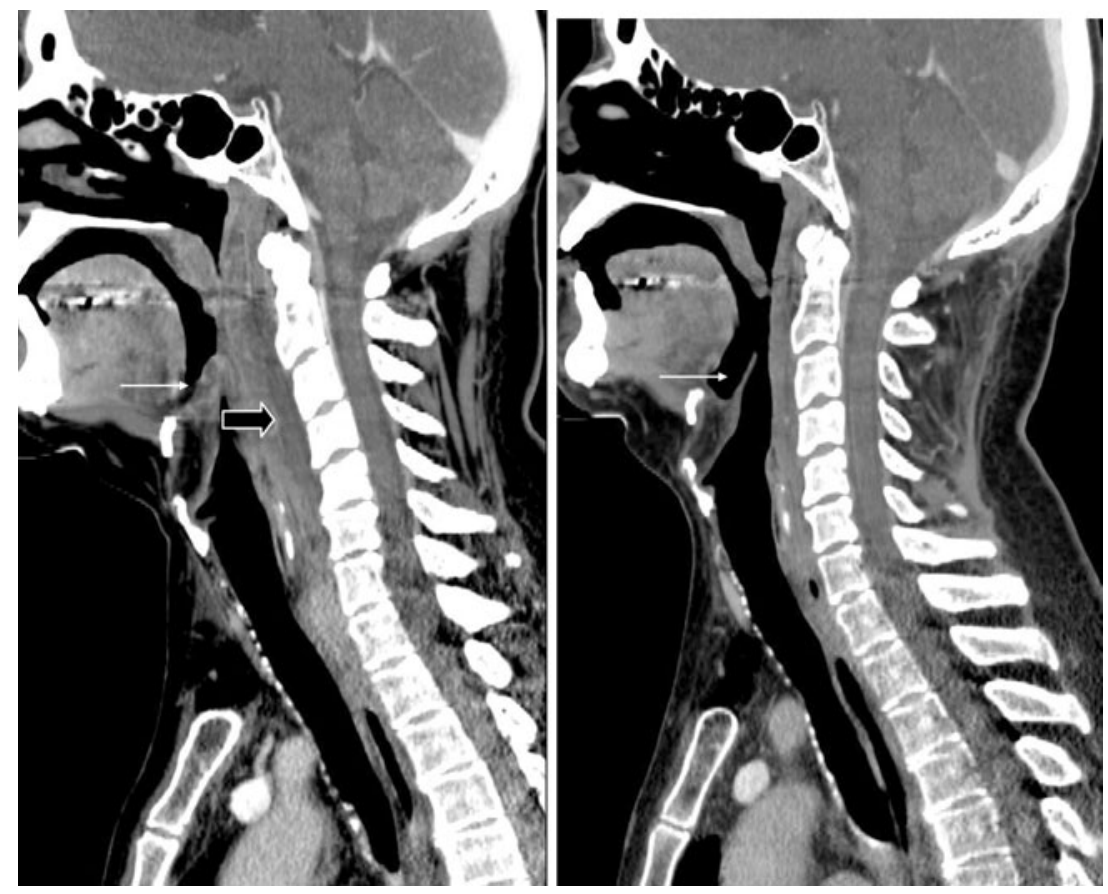

Figure 2. Two post-contrast computed tomography (CT) studies of the neck separated by 2 days. First study (a) sagittal reconstruction image showing fluid and edema in the retropharyngeal space (black arrow). Note also the enlarged epiglottis (white arrow). Subsequent study (b) with comparable image showing marked improvement. 
lymphocytes was noted. Absolute CD19+ lymphocyte count was reduced to $0.011 \times 10^{9} / \mathrm{L}$. Serum protein electrophoresis showed a markedly low gamma peak of $2.4 \mathrm{~g} / \mathrm{L}(5.0$ - 13.0). No evidence of monoclonal gammopathy was seen on serum immunofixation. IgG was $4.94 \mathrm{~g} / \mathrm{L}$ (7.51 - 15.60); IgA was <0.07 g/L (0.82 - 4.53); IgM was $0.21 \mathrm{~g} / \mathrm{L}(0.46-3.04)$. Specific antibody titers for measles, mumps, rubella, varicella, tetanus and diphtheria were all protective.

A primary immune deficiency is possible, as secondary causes resulting in impaired immunity have been excluded. However, the low albumin must be explained, as it is not a feature of a primary immunodeficiency. Profound protein loss via the gastrointestinal tract or the renal system could explain the hypogammaglobulinemia and associated low albumin. I would request a $24 \mathrm{~h}$ urine collection to exclude significant proteinuria. There has been no suggestion of liver disease or malnutrition to explain impaired protein synthesis. Protein-losing gastroenteropathy is an alternate mechanism that could explain these findings. I would inquire about abdominal pain and diarrhea. I would request a CT of the chest, abdomen and pelvis and request endoscopic investigations to exclude mucosal pathologies, such as inflammatory bowel disease and endoscopic biopsies, to exclude granulomatous or malignant involvement of the lymphatic system.

Panendoscopy and multiple biopsies revealed normal gastrointestinal histopathology. Tissue transglutaminase antibodies were negative. Stool for alpha-1 antitrypsin was not elevated. 24-hour urine protein revealed no significant proteinuria. Pneumocystis pneumonia prophylaxis was initiated with trimethoprim-sulfamethoxazole $(80 \mathrm{mg} / 400 \mathrm{mg}) 1$ tablet daily. Repeat blood work after hospital discharge showed persistence of the lymphopenia $\left(0.330 \times 10^{9}\right.$ cells $/ \mathrm{L}$, CD4 $\left.=54 \times 10^{6} / \mathrm{L}\right)$ and hypogammaglobulinemia, with recovery of the serum proteins and albumin to near normal levels.

Extensive investigations for secondary causes of hypogammaglobulinemia (e.g. nephrotic syndrome or proteinlosing enteropathy) and lymphopenia, (e.g. malignancy, drugs, HIV and other viral infections), have failed to identify a cause. This suggests that he probably has a primary immunodeficiency, likely common variable immunodeficiency (CVID). Lymphopenia can be seen in a subset of patients with CVID. Patients with CVID can also develop non-caseating granulomas, and thus a misdiagnosis of sarcoidosis is not infrequent in this setting. I would ask about a family history of immunodeficiency states and test his response to both protein and polysaccharide vaccines. He should receive intravenous immunoglobulin as part of his treatment regimen.

There was no family history for serious infections, unexplained sudden deaths, diagnosed immunodeficien- cies or autoimmune diseases. Dynamic antibody testing for multiple pneumococcal capsular antigens could not be done, as he had received pneumococcal polysaccharide vaccine 2 years earlier. A diagnosis of Common Variable Immunodeficiency (CVID) was made and intravenous immunoglobulin (IVIG) treatment was initiated. After initiation of IVIG, the lymphopenia persisted; however, he has remained infection-free 12 months after the diagnosis and has returned to fulltime work.

\section{COMMENTARY}

Clinicians rely upon clinical acumen and experience to recognize a disease in a rapid and unconscious way when the presentation appears familiar. ${ }^{1,2}$ These mental shortcuts work well in the majority of cases, but can lead to the wrong conclusion. The correct diagnosis is often not contained in the initial set of hypothesis in difficult diagnostic dilemmas. This case illustrates a complex clinical problem where the diagnosis is ultimately found by inferential (inductive) reasoning.

The original diagnosis of sarcoidosis appeared reasonable, in light of the patients' initial clinical presentation and pattern recognition by the treating clinicians. However once atypical features presented, further investigations were warranted. When fungal and then further bacterial infections were diagnosed and the hypogammaglobulinemia could not be explained by secondary causes, it became clear that a reanalysis of the case was necessary. Being able to look at the "big picture," the discussant had the advantage of being able to put the whole case in context and combine, integrate and interpret all of the data. Although a diagnosis of exclusion, CVID does appear to be the "best fit" for this patient's various clinical, histological and laboratory findings. When the clinical course deviates from the expected, and illness scripts "do not fit," then clinicians are compelled to keep searching for alternate explanations. Too often as clinicians we do the reverse, trying to 'prove' our original hypothesis. In these circumstances, the diagnosis is only revealed after extensive time and investigations have been expended, as illustrated in this complex case. Sometimes what appears to be obviously true isn't, and clinicians should be prepared to reconsider the initial diagnosis when the subsequent clinical course is unexpected.

\section{CLINICAL TEACHING POINTS}

1. Cryptococcal meningoencephalitis is often associated with advanced HIV disease or other predisposing condition, such as hematologic malignancy, sarcoidosis, solid organ transplantation or cell-mediated immune 
dysfunction. Although disease can occur in up to $20 \%$ of healthy non-immunocompromised patients, Cryptococcal meningoencephalitis remains an important opportunistic infection in patients with impaired immunity. ${ }^{3}$

2. Common variable immunodeficiency (CVID) is a disorder characterized by a failure to produce immunoglobulin and protective antibodies, due to failed B cell maturation. There is a misconception that primary immunodeficiencies are extremely rare or restricted to children. This, along with the enormous heterogeneity in clinical presentation, contributes to delays in its recognition and diagnosis. The mean age of symptom onset is 26 years, whereas the mean age of diagnosis is 35 years. $^{4}$

3. CVID is characterized by a reduction of at least two serum immunoglobulins (IgG and either IgA or $\operatorname{IgM}$ ) by more than two standard deviations from the mean. A history of multiple bacterial infections is present in $90 \%$ of patients, and up to three quarters will have additional non-infectious manifestations. ${ }^{5,6}$ CVID remains a diagnosis of exclusion. The mainstay of treatment, high-dose immunoglobulin IgG replacement, is well tolerated, effective in reducing infections, including serious life threatening infections, and improves quality of life in patients with CVID. ${ }^{4}$

4. Late-onset combined immunodeficiency, seen in about $10 \%$ of patients with CVID, is characterized and defined by the occurrence of opportunistic infection(s) and/or a CD4 T cell count of $<200 \times 10^{6}$ cells/L. ${ }^{7}$ These patients present with lymphopenia, opportunistic infections and a higher prevalence of non-caseating granulomas.

5. Granulomata form in a response to an antigenic stimulus, such as intracellular bacteria, mycobacteria and fungi, as well as non-infectious foreign matter, as in the case of pulmonary berylliosis. ${ }^{8}$ The pathogenesis of granulomatous disease in both sarcoidosis and CVID is not well understood. A misdiagnosis of sarcoidosis is not infrequent in the setting of CVID. ${ }^{9}$

Acknowledgements: We would like to thank Dr. Bruce Gray for providing and interpreting the CT and MRI images, and Dr. Jason Lee for clinical input.

Conflict of Interest: The authors declare that they do not have a conflict of interest.

\section{Financial Support: None}

Corresponding Author: Ophyr Mourad, MD, MSc; Department of Medicine, St. Michael's Hospital, University of Toronto, 30 Bond Street, Toronto, ON, Canada M5B 1W8 (e-mail: mourado@smh.ca).

\section{REFERENCES}

1. Kempainen RR, Migeon MB, Wolf FM. Understanding our mistakes: a primer on errors in clinical reasoning. Med Teach. 2003;25:177-181.

2. Eva KW. What every teacher needs to know about clinical reasoning. Med Education. 2007;39:98-106.

3. Pappas PG, Perfect JR, Cloud GA, et al. Cryptococcosis in human immunodeficiency virus-negative patients in the era of effective azole therapy. Clin Infect Dis. 2001;33:690-699.

4. Chapel H, Cunningham-Rundles C. Update in understanding common variable immunodeficiency disorders (CVIDs) and the management of patients with these conditions. Br J Haematol. 2009; 145:709-727.

5. Agarwal S, Cunningham-Rundles $\mathbf{C}$. Autoimmunity in common variable immunodeficiency. Curr Allergy Asthma Rep. 2009;9:347-352.

6. Cunningham-Rundles $\mathbf{C}$, Bodian $\mathbf{C}$. Common variable immunodeficiency: clinical and immunological features of 248 patients. Clin Immunol. 1999;92:34-48.

7. Malphettes M, Gérard L, Carmagnat M, Mouillot G, Vince N, Boutboul D, et al. Late-onset combined immune deficiency: a subset of common variable immunodeficiency with severe $\mathrm{T}$ cell defect. Clin Infect Dis. 2009;49(9):1329-1338.

8. Janeway CA, Travers $\mathbf{P}$, Walport $\mathbf{M}$, et al. Immunobiology: The immune system in health and disease. Garland Science, 2001.

9. Arnold DF, Wiggins J, Cunningham-Rundles C, Misbah SA, Chapel HM. Granulomatous disease: distinguishing primary antibody disease from sarcoidosis. Clin Immunol. 2008;128:18-22. 